\title{
Mathematics Today \\ Twelve Informal Essays
}




\title{
Mathematics Today \\ Twelve Informal
}

Essays

\author{
Edited by \\ Lynn Arthur Steen
}

Springer-Verlag

New York Heidelberg Berlin 
Dr. Lynn A. Steen

Saint Olaf College

Northfield, Minnesota 55057

USA

AMS Subject Classification: 00A05, 00A10, 00A25

Library of Congress Cataloging in Publication Data

Main entry under title:

Mathematics today.

CONTENTS: Graham, R. L. Combinatorial scheduling

theory.-Moore, D. S. Statistical analysis of experi-

mental data.-Davis, M. What is a computation? [etc.]

1. Mathematics-Addresses, essays, lectures.

I. Steen, Lynn A., 1941-

QA7.M3447 $510 \quad 78-7594$

This material was prepared with the support of National Science Foundation Grant OSS-7526057. However, any opinions, findings, conclusions, or recommendations expressed therein are those of the authors and do not necessarily reflect the views of the NSF.

All rights reserved.

No part of this book may be translated or reproduced in any form without written permission from the copyright holder.

(C) 1978 by the Conference Board of the Mathematical Sciences.

Softcover reprint of the hardcover lst edition 1978

\section{1}




\section{Preface}

The objective of the present book of essays is to convey to the intelligent nonmathematician something of the nature, development, and use of mathematical concepts, particularly those that have found application in current scientific research. The idea of assembling such a volume goes back at least to 1974, when it was discussed by the then-newly-formed Joint Projects Committee for Mathematics (JPCM) of the American Mathematical Society, the Mathematical Association of America, and the Society for Industrial and Applied Mathematics. Currently, the nine members of the JPCM are Saunders Mac Lane (Chairman) of the University of Chicago, Frederick J. Almgren, Jr. of Princeton University, Richard D. Anderson of Louisiana State University, George E. Carrier of Harvard University, Hirsh G. Cohen of the International Business Machines Corporation, Richard C. DiPrima of Rensselaer Polytechnic Institute, Robion C. Kirby of the University of California at Berkeley, William H. Kruskal of the University of Chicago, and George D. Mostow of Yale University.

The JPCM decided to make production of this volume its first major project and requested the Conference Board of the Mathematical Sciences (CBMS), of which its three sponsoring societies are all member organizations, to approach the National Science Foundation on its behalf for support of the undertaking. A proposal submitted by the CBMS in December 1974 and in revised form in July 1975 was granted by the Foundation in May 1976, and work on assembling the volume got under way. Direction of the project has been carried out by a five-member Steering Committee formed for this purpose by the JPCM. The Steering Committee has consisted of Paul R. Halmos of the University of California at Santa Barbara, Allen L. Hammond of Science magazine, Jack C. Kiefer of Cornell University, Harry Schwartz of the New York Times, and Jacob T. Schwartz of New 
York University (Chairman). Throughout the course of operations, Lynn A. Steen of St. Olaf College has served as Editor and coordinator, while CBMS headquarters has provided administrative management.

The Steering Committee would like to acknowledge the contributions of all those who have been involved in this essay project. First of all, it is to the individual essay authors that prime credit will be due for whatever success the volume achieves. All who served on the JPCM while the project was in progress deserve thanks for their helpful advice and support. Lynn Steen's able and energetic work throughout as Editor and coordinator was invaluable, as was the role of the CBMS in securing the grant for the project and managing operations under it. The publisher, Springer-Verlag, specifically its production and editorial staff, has cooperated in admirable fashion in the production of the volume. Finally, for itself and on behalf of the JPCM and the CBMS, the Steering Committee would like to express deep appreciation to the National Science Foundation and its Office of Public Understanding of Science for support of this project. 


\section{Contents}

Preface v v v v

Mathematics Today / Lynn Arthur Steen 1

$\begin{array}{lr}\text { Part One } & 13\end{array}$

Mathematics-Our Invisible Culture / Allen L. Hammond 15

$\begin{array}{ll}\text { Part Two } & 35\end{array}$

Number Theory / lan Richards 37

Groups and Symmetry / Jonathan Alperin 65

The Geometry of the Universe / Roger Penrose 83

$\begin{array}{ll}\text { The Mathematics of Meteorology / Philip Thompson } & 127\end{array}$

The Four Color Problem / Kenneth Appel and 153

Wolfgang Haken

$\begin{array}{ll}\text { Part Three } & 181\end{array}$

Combinatorial Scheduling Theory / Ronald Graham 183

Statistical Analysis of Experimental Data / David S. Moore 213

What is a Computation? / Martin Davis 241 
viii Contents

Mathematics as a Tool for Economic

Understanding / Jacob Schwartz

Mathematical Aspects of Population

Biology / Frank C. Hoppensteadt

Part Four

The Relevance of Mathematics / Felix E. Browder and Saunders Mac Lane

Further Reading

Index 